Relations industrielles

Industrial Relations

\title{
Thuderoz, Christian, Négociations : essai de sociologie du lien social
}

\section{Jean Sexton}

Volume 55, numéro 4, 2000

URI : https://id.erudit.org/iderudit/051366ar

DOI : https://doi.org/10.7202/051366ar

Aller au sommaire du numéro

Éditeur(s)

Département des relations industrielles de l'Université Laval

ISSN

0034-379X (imprimé)

1703-8138 (numérique)

Découvrir la revue

Citer ce compte rendu

Sexton, J. (2000). Compte rendu de [Thuderoz, Christian, Négociations : essai de sociologie du lien social]. Relations industrielles / Industrial Relations, 55(4), 789-790. https://doi.org/10.7202/051366ar

Tous droits réservés (C Département des relations industrielles de l'Universite Laval, 2000
Ce document est protégé par la loi sur le droit d'auteur. L’utilisation des services d'Érudit (y compris la reproduction) est assujettie à sa politique d'utilisation que vous pouvez consulter en ligne.

https://apropos.erudit.org/fr/usagers/politique-dutilisation/ 
pluridisciplinaire sur la notion de confiance ne soit jamais situé par rapport aux autres grands paradigmes explicatifs. S'agit-il d'une variante fonctionnaliste ? Comment situer la " confiance " eu égard aux analyses régulationnistes ou classistes ? Comment justifier théoriquement l'utilisation de la notion de confiance pour analyser les relations sociales les plus diverses, souvent asymétriques, allant de l'interindividualité à des groupes sociaux en rapport de pouvoir? Le lecteur n'aura pas droit à ces explications. S'il parvient jusqu'à la fin du périple, il sera confondu par toutes les catégorisations de confiance mises de l'avant (souvent astuces terminologiques) et se demandera dans quelle mesure la confiance projette un prisme éclairant sur les relations sociales. Tout au long de ce livre, on aura noté que le changement et l'innovation sont tenus pour acquis (fort bien ! Mais qu'en estil de ce qui ne change pas, de ce qui se reproduit, ou des résurgences du passé ?).

Un chapitre conclusif, que Christian Thuderoz aurait pu écrire sur la base de son élan introductif, aurait certainement pu dissiper quelques confusions et mettre un peu d'ordre. Dans son état, ce livre peut être utile comme référence, en particulier les contributions de Christian Thuderoz, André Billette, Guy Bellemare et Louise Briand.

Mona-Josée GaGnon Université de Montréal

Négociations : essai de sociologie du lien social

par Christian Thuderoz, Paris : Presses universitaires de France, 2000, 290 p., ISBN 2-13-050640-2.

Rares sont les ouvrages en langue française qui s'attardent aux fondements, à la nature, aux exigences et aux implications de la négociation tant comme activité d'échange ou de négoce que comme activité sociale de détermination collective de règles. Ce livre est alors fort bienvenu en ce qu'il sera très utile tant aux étudiants et observateurs de cette activité sociale qu'aux acteurs mêmes qui y participent.

La thèse de Thuderoz peut se résumer comme suit : seule la négociation peut accroître les interdépendances, favoriser l'innovation, produire une meilleure «condensation sociale» (p. 30). Autrement dit, seule la négociation, comme activité sociale de résolution des litiges et de décision conjointe, est de nature à régénérer le lien social, redonner du sens aux démarches et aux intérêts collectifs, confirmer l'autonomie du Sujet, réintroduire de la règle cérémonielle là où le marché a désenchanté le monde (p. 40).

Thuderoz démontre de façon éloquente que la négociation, comme acti- vité sociale, est non seulement nécessaire, mais également inévitable. D'où l'intérêt de se pencher sur ce livre et ainsi revenir aux principes de base et aux fondements mêmes de cette activité qu'est la négociation.

L'auteur établit le plan de son ouvrage en fonction de la définition qu'il adopte de la négociation (c'est-à-dire un mode de résolution, une procédure d'échange et de partage, un système de décision, une technique de régulation, un moyen d'innovation et de création sociale et un processus de communication) et des deux types analytiques de négociation qu'il distingue, soit la négociation mesurée par l'intérêt et la négociation orientée vers la morale.

Fort de cette approche, Thuderoz présente son ouvrage en deux parties. La première s'attarde à la négociation mesurée par l'intérêt en l'examinant sous l'angle, successivement, du conflit, du compromis et de la décision (chap. 1, 2 et 3 de la première partie). La seconde, consacrée à la négociation orientée vers la morale, présente trois chapitres : de 
la règle, de l'ordre négocié et de la morale.

Cet ouvrage est à la fois sociologique et philosophique. L'auteur, à travers un examen fort érudit de l'expérience humaine en négociation, propose en bout de ligne un ordre toujours négocié, comme garantie de sa stabilité et de son renouvellement : la négociation, inlassablement, crée et détruit des règles.

Ce livre ne veut aucunement enseigner comment négocier. Ce n'est pas à cet égard un livre de recettes comme trop souvent en voit-on, hélas ! Thuderoz s'arrête plutôt à répondre à une question fondamentale : pourquoi négocier autant en contexte d'échange que de détermination de règles.

Il s'agit donc d'un ouvrage d'un niveau d'abstraction plus élevé que ce à quoi nous sommes habituellement confrontés en matière de négociation. Cependant, sans tomber dans le pragmatisme, il faut déplorer ce peu de place que Thuderoz réserve au concept de « pouvoir » dans son étude sur la négociation. Non seulement cette notion de pouvoir de négociation est au cœur même de la nature des échanges inhérents à toute activité de négociation, mais elle dirige également à priori, pour se consolider ou se réhabiliter, les processus pratiques par lesquels la négociation se concrétise, à savoir les structures et les stratégies de négociation. Il s'agit là d'une inévitable trilogie interdépen- dante dont le résultat même de toute négociation dépend. Peut-être faudraitil y revenir.

Un autre reproche, plus de forme, concerne l'organisation de l'ouvrage. Il est rare que, dans un même livre, on retrouve trois « chapitre $1 »$, deux «chapitre 2 » et deux « chapitre 3 » (voir le sommaire). Même l'auteur y perd son latin en se référant, dans la présentation de son ouvrage (p. 44), aux chapitres 2 , $3,4,5,6$ et 7 . On ne retrouve pas les quatre derniers.

Maintenant, les fleurs. Au-delà des reproches ici adressés, cet ouvrage de Thuderoz est d'un français impeccable, à lecture agréable et fluide. Ses nombreuses références et citations démontrent bien qu'il possède son sujet. I1 réussit à rendre son message d'une façon sublime. Ce livre devrait être lu et médité par tous ceux qui s'intéressent à la négociation. Il comble un vide.

Les relations industrielles entendues dans un sens très large sont autant techniques que valeurs. Elles performent relativement bien au niveau des techniques. Elles sont très faibles au niveau des valeurs. Et les relations industrielles exigent la compréhension de ses fondations et de ses sources intellectuelles. C'est dans ce créneau que se situe l'excellente contribution de Christian Thuderoz.

Jean SeXton Université Laval

\section{Ravenswood: The Steelworkers' Victory and the Revival of American Labor by Tom JuRAVICH and Kate BRoNFENBRENNER, Ithaca, N.Y.: ILR Press, 1999, 245 pp., ISBN 0-8014-3633-8 (alk. paper).}

Midway through the twenty-month lockout of workers at the Ravenswood Aluminum Corporation (RAC), one of the woman activists said, "I don't feel like we're fighting for ourselves. I feel like we're fighting this for every working person in America" (p. 131).
Marge Flanigan was right. The heroic battle of the United Steelworkers of America (USWA) at Ravenswood was a turning point in U.S. labour's fightback against decades of corporate union-busting tactics and the impact of economic restructuring. This battle 